\title{
PROPRIEDADES DE CHAPAS TIPO OSB, FABRICADAS COM PARTÍCULAS ACETILADAS DE MADEIRAS DE Eucalyptus grandis, Eucalyptus urophylla, Eucalyptus cloeziana E Pinus elliottii ${ }^{1}$
}

\author{
Carla Priscilla Távora Cabral ${ }^{2}$, Benedito Rocha Vital ${ }^{3}$, Ricardo Marius Della Lucia ${ }^{3}$, Alexandre Santos \\ Pimenta $^{3}$, Carlos Pedro Boechat Soares ${ }^{3}$ e Ana Márcia Macedo Ladeira Carvalho ${ }^{4}$
}

\begin{abstract}
RESUMO - Este trabalho teve como objetivo avaliar as propriedades de chapas de Oriented Strand Board (OSB), fabricadas com flocos de madeira de Eucalyptus grandis, Eucalyptus urophylla e Eucalyptus cloeziana e oriundas dos Municípios de Ponte Alta e Três Marias, no Estado de Minas Gerais. As massas específicas básicas das três espécies de eucaliptos das duas regiões foram, respectivamente: Ponte Alta $\left(0,55 ; 0,61 ; \mathrm{e} 0,70 \mathrm{~g} / \mathrm{cm}^{3}\right)$ e Três Márias $\left(0,56 ; 0,58\right.$; e $\left.0,69 \mathrm{~g} / \mathrm{cm}^{3}\right)$. Quando necessário, para manter as massas específicas das chapas próximas de $0,70 \mathrm{~g} / \mathrm{cm}^{3}$ foram acrescentadas às partículas de madeira de eucalipto partículas de madeira de Pinus elliottii, oriundo da cidade de Viçosa, com massa específica de $0,45 \mathrm{~g} / \mathrm{cm}^{3}$. Os flocos foram gerados nas dimensões médias de 90,00 × 20,00 x 0,46 mm. O adesivo utilizado foi o fenol-formaldeído, na proporção de $8 \%$ de sólidos, em relação à massa seca de partículas. Parte dos flocos de eucaliptos foram acetilados. As chapas foram prensadas à temperatura de $170^{\circ} \mathrm{C} \mathrm{e} 32 \mathrm{kgf} / \mathrm{cm}^{2}$ de pressão. As propriedades das chapas foram determinadas segundo as normas da ABNT NBR 14810-3 (2002) e ASTM-D 1037 (1991). Os resultados foram comparados utilizando-se as normas ANSI/A - 208.1 (1993) e CSA 0437-93 (1993). As chapas contendo partículas acetiladas foram mais estáveis e adsorveram menos umidade. Na tração perpendicular, observou-se que as chapas contendo $100 \%$ de flocos acetilados apresentaram resultados inferiores ao estipulado pela norma CSA O4370/93 (1993). A resistência ao arrancamento de parafuso, módulo de ruptura (paralelo e perpendicular) e compressão longitudinal (perpendicular), foi reduzida pela acetilação nas chapas contendo $100 \%$ dos flocos acetilados. As espécies que apresentaram, numericamente, as maiores médias para resistência mecânica foram: Eucalyptus grandis não acetilado (dureza Janka) e Eucalyptus cloeziana misturado com Pinus sp (módulo de ruptura). Somente a resistência à compressão longitudinal foi afetada pela região de origem da madeira. Os painéis fabricados com madeira de Eucalyptus urophylla, oriunda do Município de Três Marias, tiveram médias inferiores aos das chapas feitas com a mesma espécie, porém oriundas do Município do Rio Doce.
\end{abstract}

Palavras-chave: OSB, Eucalyptus grandis, Eucalyptus urophylla, Eucalyptus cloeziana, Pinus elliottii e acetilação.

\section{PROPERTIES OF OSB MANUFACTURED WITH WOOD STRANDS OF Eucalyptus grandis, Eucalyptus urophylla, Eucalyptus cloeziana AND Pinus elliottii}

\begin{abstract}
The objectives of this work was to evaluate the properties of OSB (Oriented Strand Board). Wood was collected from Eucalyptus grandis, Eucalyptus urophylla and Eucalyptus cloeziana on the municipalities of Ponte Alta and Três Marias, in the state of Minas Gerais. Wood densities were respectively: Ponte Alta
\end{abstract}

\footnotetext{
${ }^{1}$ Recebido em 26.09.2005 e aceito para publicação em 05.04.2006.

${ }^{2}$ Programa de Pós-Graduação em Ciência Florestal da UFV.

${ }_{3}^{3}$ Departamento de Engenharia Florestal da UFV, Viçosa-MG. E-mail: <vital@ufv.br>.

${ }^{4}$ Departamento de Engenharia Florestal da UFVJM. E-mail: <ana.marcia@ufv.br>.
} 
$\left(0.55,0.61\right.$ and $\left.0.70 \mathrm{~g} / \mathrm{cm}^{3}\right)$ and Três Marias $\left(0.56,0.58\right.$ e $\left.0.69 \mathrm{~g} / \mathrm{cm}^{3}\right)$. Strands of Pinus elliottii, derived from the city of Viçosa, with density of $0.45 \mathrm{~g} / \mathrm{cm}^{3}$, were added to strands of eucalyptus when necessary to keep board densities close to $0.70 \mathrm{~g} / \mathrm{cm}^{3}$. Flakes were fabricated in a laboratory disk flaker measuring approximately $20.00 \times 0.46 \times 90.00 \mathrm{~mm}$. Phenol-formaldehyde adhesive was applied at $8 \%$ solid rate, in relation to particle dry mass. Part of the treatment was carried out with flakes from acetylated eucalyptus. The boards were pressed at $170^{\circ} \mathrm{C}$ and $32 \mathrm{kgf} / \mathrm{cm}^{2}$ pressure. Boards properties were determined according to the ABNT NBR 14810 3 (2002) and ASTM-D 1037 (1991) norms. The results were compared using the ANSI/A - 208.1 (1993) and CSA 0437-93 (1993) norms. OSB manufactured with any amount of acetylated flakes was more stable and absorbed less water. However the resistance to perpendicular traction of OSB with $100 \%$ of acetylated flakes was inferior to the stipulated by the CSA 0437-0/93 (1993) norm. It was also found that acetylation had a negative effect on screw withdrawal resistance, module of rupture (parallel and perpendicular) and longitudinal compression resistance (perpendicular) of Eucalyptus grandis OSB having 100\% of their flakes acetylated. Boards that presented numerically the highest means were manufactured with non-acetylated flakes of Eucalyptus grandis (Janka hardness) and Eucalyptus cloeziana mixed with Pinus sp (module of rupture). Only resistance to longitudinal compression was affected by the wood origin. OSB manufactured with Eucalyptus urophylla wood, from the Cerrado, had lower means than OSB made of the same species, however, from Vale do Rio Doce.

Keywords: OSB, Eucalyptus grandis, Eucalyptus urophylla, Eucalyptus cloeziana, Pinus elliottii, acetylation.

\section{INTRODUÇÃO}

Oriented Strand Board (OSB) é um painel reconstituído de flocos de madeira, parcialmente orientados, com a incorporação de adesivo à prova d'água e consolidados por meio de prensagem a quente. As dimensões dos flocos podem variar de $25 \mathrm{~mm}$ de largura por 80 a $150 \mathrm{~mm}$ de comprimento, podendo estar dispostos na camada interna, perpendicularmente, às camadas externas, ou de forma aleatória. As chapas de OSB são produtos utilizados para aplicações estruturais, como suportes de piso e forro, componentes de vigas, estrutura de móveis, embalagens etc., competindo com o compensado. Por ser menos exigente do que o compensado na qualidade da matéria prima, o OSB está substituindo o mesmo em muitas aplicações (CLOUTIER, 1998). Para a fabricação do OSB é possível utilizar madeiras de qualidade inferior e até resíduos florestais, desde que estes apresentem dimensões que permitam a confecção dos flocos, por exemplo costaneiras. Comparando o custo de produção e preço final no mercado de produtos de madeira reconstituída, percebe-se que, atualmente, o OSB é mais econômico do que o compensado. Contudo, o compensado é um painel mais estável, e os métodos propostos para a estabilização dimensional de painéis OSB ainda não são aplicados em nível industrial, em razão dos custos e dos aspectos técnicos e operacionais (MENDES, 2001).
No Brasil, as chapas de OSB começaram a ser produzidas a partir de 2002 utilizando madeira de pinus. Contudo, a crescente demanda pela madeira de pinus tem motivado pesquisas com outras espécies, principalmente eucalipto, para suprir a indústria de chapas de partículas.

Segundo Moslemi (1974), Maloney (1993), Kelly (1977) e Hrázský e Král (2003), entre os requisitos na escolha da matéria-prima para a fabricação de chapas de madeira reconstituída, devem-se considerar densidade, estabilidade dimensional, acidez e capacidade-tampão. A densidade é uma das propriedades mais importantes, pois afeta a densidade do painel e as suas propriedades mecânicas. Madeiras de baixa densidade permitem razões de compactação mais elevadas, o que aumenta a superfície de contato entre as partículas de madeira, melhorando a adesão entre elas. Além do mais, madeiras de baixa densidade geram painéis com maior uniformidade, que possuem alta capacidade de distribuição de forças entre os flocos, melhorando suas propriedades de resistência à flexão estática e à ligação interna.

Segundo Moslemi (1974) e Maloney (1993), madeiras com densidade de até $0,55 \mathrm{~g} / \mathrm{cm}^{3}$ são as mais recomendadas para a produção de chapas de partículas. Assim, Iwakiri et al. (2004) concluíram que entre as espécies estudadas as madeiras de Eucalyptus grandis e Eucalyptus saligna, espécies com menores densidades, 
mostraram-se promissoras para a fabricação de chapas de OSB. Gouveia et al. (2000), comparando propriedades de chapas de madeira aglomerada orientadas e nãoorientadas fabricadas com partículas de madeira de Eucalyptus grandis e Eucalyptus urophylla, concluíram que aquelas fabricadas com madeira de Eucalyptus grandis, de menor densidade, foram melhores. Contudo, segundo Vital et al. (1974) é possível misturar espécies de elevada e baixa densidades para se obter uma densidade média da matéria-prima adequada à fabricação das chapas de partículas.

Madeiras com elevada variação dimensional, como é o caso das madeiras de eucalipto, podem, no entanto, ocasionar tensões nos locais de adesão que contribuem para a desagregação das chapas (KELLY, 1973). Além disso, a variação dimensional pode ultrapassar o valor máximo permitido para a comercialização das chapas, conforme observado por Gouveia et al. (2003). Contudo, a estabilidade dimensional pode ser melhorada, alterandose quimicamente a madeira (ROSENQVIST, 2001).

Uma das modificações químicas da madeira mais estudada é a acetilação que tem sido pesquisada de forma universal desde 1945. A acetilação bloqueia os grupos hidroxílicos, impedindo a formação de ligações de hidrogênio com a molécula de água, reduzindo a higroscopicidade (ROWELL, 2005) da madeira, tornandoa dimensionalmente mais estável (VICK e ROWELL, 1990; FEIST et al., 1991). Contudo, a acetilação pode reduzir a adesão entre as partículas de madeira prejudicando algumas propriedades mecânicas das chapas (OKINO et al., 1997; VICK e ROWELL, 1990; ROWELL etal., 1987; YOUNGQUIST e ROWELL, 1990). No entanto, segundo Rowell (2005) a redução na unidade de equilíbrio higroscópico das chapas de compósitos pode contribuir para que ocorra apenas pequena alteração nas propriedades das chapas de compósitos.

Assim, este trabalho teve como objetivo determinar as propriedades de chapas de OSB fabricadas com flocos de Eucalyptus grandis, Eucalyptus cloeziana e Eucalyptus urophylla, às quais foram adicionados flocos de madeira de Pinus elliottii, para se obterem chapas com a mesma densidade, além de determinar o efeito da acetilação na estabilidade dimensional e demais propriedades das chapas.

\section{MATERIAIS E MÉTODOS}

As madeiras dos eucaliptos: Eucalyptus grandis, Eucalyptus cloeziana e Eucalyptus urophylla foram obtidas de plantios comerciais das empresas CAF, Gerdau e Plantar, na região do Vale do Rio Doce (Município de Ponte Alta) e na região de Cerrado (Município de Três Marias), no Estado de Minas Gerais. A madeira de Pinus elliottii foi coletada no campus da Universidade Federal de Viçosa, em Viçosa, MG. Foram colhidas cinco árvores por espécie em cada região. A idade das árvores de Eucalyptus grandis colhida na região do Vale do Rio Doce era de 10 anos. As demais árvores tinham 7 anos de idade.

Inicialmente, todas as toras foram desdobradas em tábuas, com $1 \mathrm{~m}$ de comprimento e $2 \mathrm{~cm}$ de espessura. As tábuas e costaneiras foram novamente serradas, produzindo-se baguetas com $2 \mathrm{~cm}$ de espessura e 9 $\mathrm{cm}$ de largura. Após a saturação em água, as baguetas foram processadas num moinho de disco, produzindose flocos com as seguintes dimensões, $90 \mathrm{~mm}$ x $20 \mathrm{~mm}$ x $0,46 \mathrm{~mm}$.

Para separar os finos, empregou-se uma peneira com malha quadrangular e área do orifício de 179,94 $\mathrm{mm}^{2}$. Após essa seleção, os flocos foram secados em estufa a uma temperatura de $70{ }^{\circ} \mathrm{C}$ e ventilação forçada até atingir o teor de umidade aproximadamente $3 \%$. Em seguida, foram armazenados em sacos plásticos até a fabricação das chapas.

A acetilação seguiu o procedimento adotado por Rowell et al. (1990). Os flocos de eucalipto foram secados em estufa aquecida a $100 \pm 3^{\circ} \mathrm{C}$, com ventilação forçada por um período de 24 horas. Logo em seguida, foram pesados e colocados em uma autoclave eletrônica e saturados, por uma hora, com anidrido acético.

$\mathrm{O}$ anidrido foi drenado e a autoclave aquecida a $120^{\circ} \mathrm{C}$, permanecendo, assim, por $3 \mathrm{~h}$. Os flocos foram deixados por mais duas horas na autoclave, sob efeito de vácuo. Depois dessa etapa, eles foram retirados, deixados ao ar livre por $24 \mathrm{~h}$ e levados novamente para a estufa, onde ficaram por mais $24 \mathrm{~h}$, para serem, em seguida, novamente pesados. O teor de acetilação foi determinado pela diferença de massa antes e depois do tratamento.

Para a confecção das chapas foi utilizado o adesivo fenol-formaldeído (CASCOPHEN HL, 2080), contendo $48 \%$ de sólidos resinosos, doados pela Empresa Alba Química e aplicados na proporção de $8 \%$ em relação à quantidade de massa seca de partículas. Também foi aplicada uma solução de parafina $0,5 \%$, em todos

R. Árvore, Viçosa-MG, v.30, n.4, p.659-668, 2006 
os tratamentos. Tanto o adesivo quanto a parafina foram aplicados em um encolador de tambor, dotado de uma pistola de pintura acionada por ar comprimido.

Para se determinar a massa específica, foram selecionadas, aleatoriamente, 10 baguetas por espécie e origem da madeira. Empregou-se o método de imersão em água, conforme descrito por Vital (1984).

A quantidade de flocos de cada espécie foi calculada de forma a se obterem chapas com densidade igual a $0,70 \mathrm{~g} / \mathrm{cm}^{3}$.

Para a formação do colchão, os flocos das faces foram orientados perpendicularmente à camada interna, na proporção de $25 \%$ do total dos flocos, em cada face e $50 \%$ do total dos flocos na constituição da camada interna. Para a orientação dos flocos, foi utilizada uma grade de ferro com várias divisórias paralelas, conforme mostrado na Figura 1.

As chapas, medindo cerca de $40 \mathrm{~cm} \mathrm{x} 40 \mathrm{~cm} \times 1$ $\mathrm{cm}$ foram prensadas a uma temperatura de $170{ }^{\circ} \mathrm{C} \mathrm{e}$ uma pressão de $32 \mathrm{kgf} / \mathrm{cm}^{2}$, por um período de $8 \mathrm{~min}$, contados a partir do fechamento da prensa.

Os corpos-de-prova foram climatizados até o equilíbrio, a uma umidade relativa de $65 \pm 5 \%$ e a uma temperatura de $20 \pm 3{ }^{\circ} \mathrm{C}$, conforme estabelecido pela norma ABNT NBR 14810-3 (2002).

Todos os ensaios foram realizados conforme a norma da ABNT: NBR 14810-3 (2002) e os valores obtidos nos testes foram comparados com a norma ANSI/A - 208.1 (1993) e a norma canadense CSA O437-93.
Para determinar a expansão linear, o inchamento em espessura e a adsorção de vapor de água, os corposde-prova foram novamente climatizados até equilíbrio à temperatura de $20 \pm 3{ }^{\circ} \mathrm{C}$ e umidade relativa de $90 \pm 5 \%$, conforme estebelece a norma ASTM-D 1037, 1991.

O experimento foi constituído de 12 tratamentos com duas repetições, totalizando 24 chapas, variando o tipo de partícula (flocos acetilados e flocos nãoacetilados), espécie e procedência. A porcentagem de flocos de Pinus elliottii adicionada aos flocos de madeira de eucalipto, bem como a procedência das madeiras de eucalipto, e mostrada no Quadro 1.

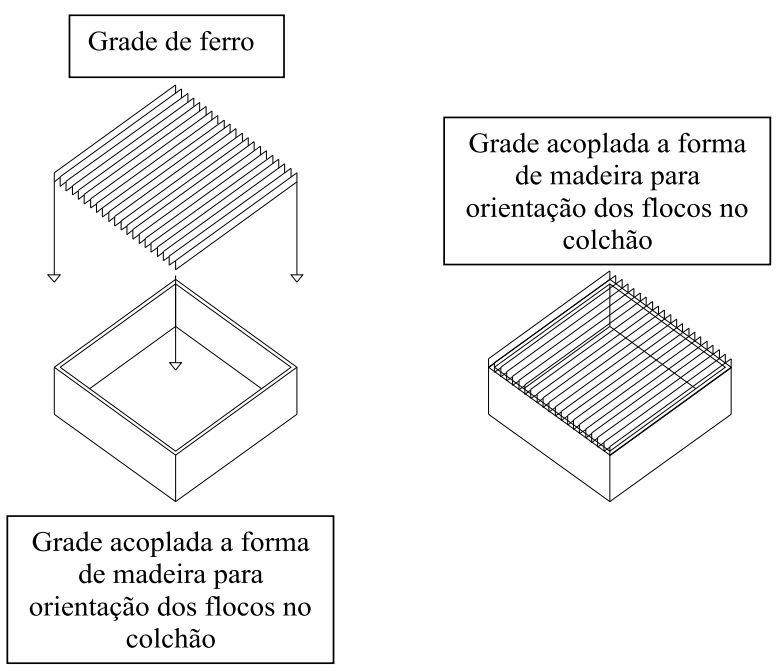

Figura 1 - Ilustração da caixa e da grade formadora do colchão. Figure 1 -Mat forming and strand orientation device.

Quadro 1 - Composição dos flocos e procedência das madeiras de eucaliptos Table 1 - Flake composition and wood provenance

\begin{tabular}{|c|c|c|c|c|c|}
\hline \multirow[t]{2}{*}{ Tratamento } & \multirow[t]{2}{*}{ Tipo de Flocos } & \multirow[t]{2}{*}{ Espécie } & \multicolumn{2}{|c|}{ Composição das Partículas(\%) } & \multirow[t]{2}{*}{ Procedência } \\
\hline & & & Pinus & Eucalipto & \\
\hline $\mathrm{T} 1$ & Não acetilados & Eucalyptus grandis & 0 & 100 & Região 1 \\
\hline $\mathrm{T} 2$ & Não acetilados & Eucalyptus urophylla & 40 & 60 & Região 1 \\
\hline $\mathrm{T} 3$ & Não acetilados & Eucalyptus cloeziana & 60 & 40 & Região 1 \\
\hline $\mathrm{T} 4$ & Não acetilados & Eucalyptus grandis & 0 & 100 & Região 2 \\
\hline $\mathrm{T} 5$ & Não acetilados & Eucalyptus urophylla & 10 & 90 & Região 2 \\
\hline T 6 & Não acetilados & Eucalyptus cloeziana & 55 & 45 & Região 2 \\
\hline $\mathrm{T} 7$ & Acetilados & Eucalyptus grandis & 0 & 100 & Região 1 \\
\hline $\mathrm{T} 8$ & Acetilados & Eucalyptus urophylla & 40 & 60 & Região 1 \\
\hline T 9 & Acetilados & Eucalyptus cloeziana & 60 & 40 & Região 1 \\
\hline $\mathrm{T} 10$ & Acetilados & Eucalyptus grandis & 0 & 100 & Região 2 \\
\hline $\mathrm{T} 11$ & Acetilados & Eucalyptus urophylla & 10 & 90 & Região 2 \\
\hline $\mathrm{T} 12$ & Acetilados & Eucalyptus cloeziana & 55 & 45 & Região 2 \\
\hline
\end{tabular}

Região 1- Região do Vale do Rio Doce, Município de Ponte Alta. Região 2- Região do Cerrado, Município de Três Marias.

R. Árvore, Viçosa-MG, v.30, n.4, p.659-668, 2006 


\section{RESULTADOS E DISCUSSÃO}

As massas específicas das espécies da região do Vale do Rio Doce foram respectivamente, Eucalyptus grandis, $0,55 \mathrm{~g} / \mathrm{cm}^{3} ;$ Eucalyptus urophylla, $0,61 \mathrm{~g} / \mathrm{cm}^{3} ;$ Eucalyptua cloeziana $0,70 \mathrm{~g} / \mathrm{cm}^{3}$; e nas mesmas três espécies coletadas na região de Cerrado, foram: $0,56 \mathrm{~g} / \mathrm{cm}^{3}, 0,58 \mathrm{~g} / \mathrm{cm}^{3} \mathrm{e} 0,69$ $\mathrm{g} / \mathrm{cm}^{3}$, respectivamente. A massa específica da madeira do Pinus elliottii foi de $0,45 \mathrm{~g} / \mathrm{cm}^{3}$.

Os teores de acetilação das espécies de eucalipto da região 1(Vale do Rio Doce) e região 2 (Cerrado) foram, respectivamente: Eucalyptus grandis (21,24 e 21,23\%), Eucalyptus urophylla (23,18 e 22,30\%) e Eucalyptus cloeziana (20 e 20,71\%), que são compatíveis com aqueles encontrados por Mahlberg et al. (2001), Rosenqvist (2001); Vick e Rowell (1990), que observaram ganho em peso variando de 17,2 a $22,1 \%$.

Pela análise de variância das médias das densidades, não se observaram diferenças significativas entre os tratamentos. Segundo a ANSI/A1-280/93, os painéis com densidade variando entre 0,60 e $0,80 \mathrm{~g} / \mathrm{cm}^{3}$ são classificados como painéis de densidade média, portanto os painéis fabricados se encaixam nessa classificação.

Os valores médios de adsorção de água (\%) constam no Quadro 2. Não houve diferença oriunda da origem das espécies, contudo se observou diferença significativa entre as médias das chapas contendo flocos acetilados e não-acetilados. A acetilação reduziu a taxa de adsorção de água em todas as espécies. Esse resultado era esperado e está de acordo com o de outros trabalhos, como de Imamura et al. (1989), que observaram reduções médias de 40 e $54 \%$ na adsorção de vapor de água nas chapas de aglomerado fabricadas com adesivo à base de fenol-formaldeído e isocianato, respectivamente, e partículas de Albizia facata. Isso ocorreu devido ao bloqueio dos sítios de adsorção pela acetilação.

A acetilação reduziu também, de forma significativa, o inchamento em espessura. Nas chapas fabricadas com flocos acetilados, não houve diferença entre regiões e espécies de madeira, conforme pode ser observado nos Quadros 3 e 4.

Entre os painéis fabricados com flocos nãoacetilados, observou, contudo, que os painéis menos estáveis foram aqueles fabricados com flocos de Eucalyptus grandis, que foi a espécie para a qual se observou maior adsorção de vapor de água. Além disso, as chapas produzidas de flocos oriundos de madeira da região 2 apresentaram inchamento ligeiramente superior àquelas fabricadas com madeiras da região 1. As maiores diferenças numéricas são observadas quando se comparam as chapas fabricadas com flocos não-acetilados.

Quadro 2 - Valores médios de adsorção de vapor de água (\%), em função da espécie e acetilação

Table 2 - Mean values of water adsorption as a function of species and acetylation

\begin{tabular}{|c|c|c|}
\hline \multirow[t]{2}{*}{ Espécies } & \multicolumn{2}{|c|}{$\begin{array}{c}\text { Valores médios de adsorção } \\
\text { de água(\%) }\end{array}$} \\
\hline & Não-acetiladas & Acetiladas \\
\hline Eucalyptus grandis & 9,17 (a)A & 1,64 (a)B \\
\hline Eucalyptus urophylla & 8,22 (b)A & 1,76 (a)B \\
\hline Eucalyptus cloeziana & 8,55 (b)A & 1,82 (a)B \\
\hline
\end{tabular}

* Médias seguidas de mesma letra minúscula numa mesma coluna ou mesma letra maiúscula na mesma linha não diferem entre si, estatisticamente, em nível de 5\% de significância, pelo teste de Tukey.

Quadro 3 - Valores médios de inchamento em espessura (\%), em função da espécie e acetilação

Table 3 - Thickness swelling mean values (\%) as a function of species and acetylation

\begin{tabular}{|c|c|c|}
\hline \multirow[t]{2}{*}{ Espécies } & \multicolumn{2}{|c|}{$\begin{array}{c}\text { Valores médios de inchamento } \\
\text { em espessura }(\%)\end{array}$} \\
\hline & Não-acetiladas & Acetiladas \\
\hline Eucalyptus grandis & 6,67 (a)A & $0,84(\mathrm{a}) \mathrm{B}$ \\
\hline Eucalyptus urophylla & 6,28 (b)A & $0,91(\mathrm{a}) \mathrm{B}$ \\
\hline Eucalyptus cloeziana & $6,44(\mathrm{ab}) \mathrm{A}$ & $0,98(a) B$ \\
\hline
\end{tabular}

* Médias seguidas de mesma letra minúscula numa mesma coluna ou mesma letra maiúscula na mesma linha não diferem entre si, estatisticamente, em nível de 5\% de significância, pelo teste de Tukey.

Quadro 4 - Valores médios de inchamento em espessura (\%) em função da região e acetilação

Table 4 - Thickness swelling mean values (\%) as a function of region and acetylation

\begin{tabular}{lcc}
\hline \multirow{2}{*}{ Regiões } & \multicolumn{2}{c}{$\begin{array}{c}\text { Valores médios de inchamento } \\
\text { em espessura }(\%)\end{array}$} \\
\cline { 2 - 3 } & Não-acetiladas & Acetiladas \\
\hline Região 1 & 6,31 (a)A & 0,92 (a)B \\
Região 2 & 6,61 (b)A & 0,90 (a)B \\
\hline
\end{tabular}

*Médias seguidas de mesma letra minúscula numa mesma coluna ou mesma letra maiúscula na mesma linha não diferem entre si, estatisticamente, em nível de 5\% de significância, pelo teste de Tukey.

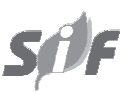

R. Árvore, Viçosa-MG, v.30, n.4, p.659-668, 2006 
A acetilação dos flocos ocasionou, ainda, redução significativa na expansão linear das chapas fabricadas com flocos das três espécies de madeira, tanto na direção paralela quanto na direção perpendicular à orientação predominante dos flocos das faces, conforme pode ser observado no Quadro 5. A expansão linear paralela foi afetada, ainda, pela origem da madeira. Os flocos não-acetilados e oriundos da região 2 resultaram em chapas menos estáveis do que aquelas oriundas da região 1. A expansão na direção perpendicular foi afetada apenas pela acetilação que, conforme esperado, reduziu, significativamente, a magnitude da expansão.

A acetilação, de modo geral, resultou em chapas com maior dureza na direção normal à superfície. Contudo, a diferença foi significativa apenas nas chapas fabricadas com flocos de madeiras de Eucalyptus urophylla e Eucalyptus cloeziana. O fato de as partículas acetiladas apresentarem teor mais baixo de umidade pode ter contribuído para maior resistência do painel à penetração da esfera no teste de dureza. Na norma ANSI/A1-280/93, o mínimo exigido para atender às especificações do mercado é de 222,5 kgf; todos os painéis apresentaram valor superior ao estipulado pela norma, conforme mostrado no Quadro 7.

Quadro 5-Valores médios de expansão linear (\%) nas direções paralela e perpendicular à orientação predominante dos flocos das faces, em função da espécie e acetilação

Table 5 - Parallel and perpendicular linear swelling (\%) as a function of species and acetylation

\begin{tabular}{lcc}
\hline \multicolumn{4}{c}{ Valores Médios de Expansão Linear (\%) } \\
\hline \multicolumn{3}{c}{ Direção Paralela } \\
\hline \multicolumn{1}{c}{ Espécies } & Não-Acetiladas & Acetiladas \\
Eucalyptus grandis & 0,069 (a)A & 0,011 (a)B \\
Eucalyptus urophylla & 0,065 (b)A & 0,010 (a)B \\
Eucalyptus cloeziana & 0,065 (b)A & 0,011 (a)B \\
\hline \multicolumn{3}{c}{ Direção Perpendicular } \\
\hline \multicolumn{1}{c}{ Espécies } & Não Acetiladas & Acetiladas \\
Eucalyptus grandis & 0,072 (a)A & $0,011($ a) B B \\
Eucalyptus urophylla & 0,069 (a)A & $0,011($ a) B \\
Eucalyptus cloeziana & 0,069 (a) A & 0,010 (a) B \\
\hline
\end{tabular}

*Médias seguidas de mesma letra minúscula numa mesma coluna ou mesma letra maiúscula na mesma linha não diferem entre si, estatisticamente, em nível de 5\% de significância, pelo teste de Tukey.
Quadro 6- Valores médios de expansão linear (\%), nas direções paralela e perpendicular à orientação predominante dos flocos das faces em função da região e acetilação

Table 6-Mean values for linear expansion parallel and perpendicular to the face strands as a function of region and acetylation

\begin{tabular}{lcc}
\hline \multicolumn{3}{c}{ Valores Médios de Expansão Linear (\%) } \\
\hline Direção perpendicular \\
\hline Regiões & Não-Acetiladas & Acetiladas \\
Região 1 & 0,064 (b)A & $0,011(\mathrm{a}) \mathrm{B}$ \\
Região 2 & $0,068(\mathrm{a}) \mathrm{A}$ & 0,011 (a)b \\
\hline \multicolumn{3}{c}{ Direção paralela } \\
\hline Regiões & Não-Acetiladas & Acetiladas \\
Região 1 & 0,069 (a)A & 0,011 (a) B \\
Região 2 & 0,070 (a)A & 0,011 (a)B \\
\hline
\end{tabular}

*Médias seguidas de mesma letra minúscula numa mesma coluna ou mesma letra maiúscula na mesma linha não diferem entre si, estatisticamente, em nível de 5\% de significância, pelo teste de Tukey.

Quadro 7 - Valores médios de dureza Janka (kgf), em função da espécie e acetilação

Table 7-Hardness (kgf) as a function of species and acetylation

\begin{tabular}{lcc}
\hline & \multicolumn{2}{c}{ Valores Médios de Dureza Janka (kgf) } \\
\cline { 2 - 3 } \multicolumn{1}{c}{ Espécies } & Não-Acetiladas & Acetiladas \\
\hline Eucalyptus grandis & 685 (a)A & 698 (a)A \\
Eucalyptus urophylla & 536 (b)B & 736 (a)A \\
Eucalyptus cloeziana & 561 (b)B & 734 (a)A \\
\hline
\end{tabular}

* Médias seguidas de mesma letra minúscula numa mesma coluna ou mesma letra maiúscula na mesma linha não diferem entre si, estatisticamente, em nível de 5\% de significância, pelo teste de Tukey.

Na resistência à tração perpendicular, as chapas fabricadas com flocos acetilados de Eucalyptus grandis apresentaram menores médias quando comparadas com as demais chapas. Os painéis que apresentaram maiores valores médios de resistência foram aqueles constituídos de madeira de Eucalyptus cloeziana com Pinus. A adesão envolve mecanismos de ligações físicas e químicas que estão correlacionados com a disponibilidade dos sítios de adsorção na madeira. Quando os sítios de ligação estão bloqueados, observa-se uma queda na taxa de adesão entre as partículas, o que ocasiona redução na resistência mecânica das chapas. Se considerar que as partículas de Eucalyptus cloeziana apresentaram um valor, inferior ao das outras duas espécies, no teor de acetilação, isso leva a crer que esse fato contribuiu diretamente para um melhor desempenho na tração perpendicular. 
Na norma CSA O437-0/93, o mínimo exigido para atender às especificações do mercado é de 3,45 kgf. Todos os painéis, com exceção dos compostos com madeira de Eucalyptus grandis acetilada, apresentaram valor superior ao estipulado pela norma, conforme mostrado no Quadro 8.

A resistência média ao arrancamento de parafuso foi igual a $156,71 \mathrm{kgf}$ e não foi observada nenhuma diferença significativa entre os tratamentos. Observouse, contudo, uma leve tendência dos resultados a serem influenciados pela adesão, com isso o tratamento que apresentou a menor média foi aquele constituído somente de flocos de Eucalyptus grandis acetilado. Todos os painéis superaram o mínimo estabelecido na norma. ANSI/A1-280/93, que é igual a 100 kgf.

Para resistência à compressão longitudinal, no sentido perpendicular à orientação predominante dos flocos da face observou-se que, dentro da mesma região, não houve diferença significativa entre as espécies. Na região 2 , no entanto, a resistência das chapas fabricadas com flocos de Eucalyptus urophylla foi significativamente menor do que aquelas fabricadas com flocos da mesma espécie, porém oriundas da região 1 , o que pode ser mais bem visualizado no Quadro 9.

O efeito dos tratamentos no módulo de ruptura em flexão estática pode ser observado nos Quadros 10 e 11. Tanto na direção paralela quanto na direção perpendicular à orientação dos flocos, observouse que a acetilação ocasionou redução significativa no módulo de ruptura das chapas fabricadas com flocos de Eucalyptus grandis. Esses resultados são coerentes com aqueles observados por Rowell et al. (1987) e Youngquist e Rowell (1990), que observaram redução de 293 para $194 \mathrm{kgf} / \mathrm{cm}^{2}$ e 344 para $266 \mathrm{kgf} /$ $\mathrm{cm}^{2}$ para chapas de flocos aspen fabricadas com $5 \%$ de adesivo fenólico e isocianato, respectivamente.

Quadro 8 - Valores médios de tração perpendicular (kgf/ $\mathrm{cm}^{2}$ ) por espécies para chapas fabricadas com flocos acetilados e não acetilados

Table 8 -Board internal bond strength as a function of species and acetylation

\begin{tabular}{lcc}
\hline \multicolumn{3}{c}{ Valores médios de tração perpendicular $\left(\mathrm{kgf} / \mathrm{cm}^{2}\right)$} \\
\hline \multicolumn{1}{c}{ Espécies } & não-acetiladas & acetiladas \\
\hline Eucalyptus grandis & 4,62(a)A & $3,24(\mathrm{~b}) \mathrm{B}$ \\
Eucalyptus urophylla & $3,60(\mathrm{a}) \mathrm{A}$ & $3,97(\mathrm{a}) \mathrm{A}$ \\
Eucalyptus cloeziana & $3,78(\mathrm{a}) \mathrm{A}$ & $4,82(\mathrm{a}) \mathrm{A}$ \\
\hline
\end{tabular}

* Médias seguidas de mesma letra minúscula numa mesma coluna ou mesma letra maiúscula na mesma linha não diferem entre si, estatisticamente, em nível de 5\% de significância, pelo teste de Tukey.
Quadro9-Valores médios de resistência à compressão longitudinal $\left(\mathrm{kgf} / \mathrm{cm}^{2}\right)$, na direção perpendicular à orientação predominante dos flocos das faces, em função das espécies e regiões de origem da madeira

Table 9-Mean compression strength parallel and perpendicular to side strands as a function of species and wood provenance

Valores Médios de Compressão Longitudinal $\left(\mathrm{kgf} / \mathrm{cm}^{2}\right)$ Direção Perpendicular

\begin{tabular}{lcc}
\hline Espécies & Região 1 & Região 2 \\
Eucalyptus grandis & 92,59 (a)A & 73,68 (a)A \\
Eucalyptus urophylla & 117,14 (a)A & 75,43 (a)B \\
Eucalyptus cloeziana & 85,82 (a)A & 101,37 (a)A \\
\hline
\end{tabular}

* Médias seguidas de mesma letra minúscula numa mesma coluna ou mesma letra maiúscula na mesma linha não diferem entre si, estatisticamente, em nível de 5\% de significância, pelo teste de Tukey.

A acetilação não afetou, de forma significativa, o modulo das chapas fabricadas com flocos de Eucalyptus urophylla e Eucalyptus cloeziana.

Observou-se ainda que a resistência, tanto no sentido paralelo quanto no perpendicular, das chapas fabricadas com flocos de Eucalyptus grandis foi inferior à resistência das chapas contendo flocos de Eucalyptus urophylla e Eucalyptus cloeziana.

Considerando a média geral, como mostrado no Quadro 11, observou-se, também, que as chapas fabricadas com flocos de Eucalyptus cloeziana e Eucalyptus urophilla foram, significativamente, mais resistentes do que aquelas fabricadas com flocos de Eucalyptus grandis.

Quadro 10 - Valores médios de módulo de ruptura $\left(\mathrm{kgf} / \mathrm{cm}^{2}\right)$, nas direções perpendicular e paralela à orientação predominante dos flocos das faces, em função das espécies e acetilação

Table 10 - Mean strand modulus of rupture parallel and perpendicular to face as a function of species and acetylation

\begin{tabular}{lcc}
\hline \multicolumn{3}{c}{ Valores Médios de Módulo de Ruptura $\left(\mathrm{kgf} / \mathrm{cm}^{2}\right)$} \\
\hline \multicolumn{3}{c}{ Direção Perpendicular } \\
\hline Espécies & Não-acetiladas & Acetiladas \\
Eucalyptus grandis & 265,06 (a)A & 177,15 (b)B \\
Eucalyptus urophylla & 227,64 (a)A & 259,65 (a)A \\
Eucalyptus cloeziana & 247,59 (a)A & 305,61 (a)A \\
\hline \multicolumn{4}{c}{ Direção Paralela } \\
\hline Espécies & Não-acetiladas & Acetiladas \\
Eucalyptus grandis & 338,45 (a)A & 229,80 (b)B \\
Eucalyptus urophylla & 313,00 (a)A & 335,62 (a)A \\
Eucalyptus cloeziana & 314,87 (a)A & 371,94 (a)A \\
\hline
\end{tabular}

*Médias seguidas de mesma letra minúscula numa mesma coluna ou mesma letra maiúscula na mesma linha não diferem entre si, estatisticamente, em nível de 5\% de significância, pelo teste de Tukey. 
Quadro 11 - Valores médios de módulo de ruptura $\left(\mathrm{kgf} / \mathrm{cm}^{2}\right)$, em função das espécies

Table 11 - Modulus of rupture mean values as a function of species

\begin{tabular}{lc}
\hline \multicolumn{2}{c}{ Valores Médios de Módulo de Ruptura $\left(\mathrm{kgf} / \mathrm{cm}^{2}\right)$} \\
\hline Espécies & Média geral \\
\hline Eucalyptus grandis & 284 (b) \\
Eucalyptus urophylla & 324 (a) \\
Eucalyptus cloeziana & 343 (a) \\
\hline
\end{tabular}

* Médias seguidas da mesma letra minúscula ao longo da coluna não diferem entre si, estatisticamente, em nível de 5\% de significância, pelo teste de Tukey.

A norma CSA O437-0/93 estipula um valor mínimo de $290 \mathrm{kgf} / \mathrm{cm}^{2}$ para o módulo de ruptura paralelo à orientação predominante dos flocos das faces e outro de $124 \mathrm{kgf} /$ $\mathrm{cm}^{2}$ para o módulo de ruptura no sentido perpendicular. Portanto, todas as chapas tiveram resistência superior ao valor mínimo estabelecido pela norma.

Não se observou diferença significativa no módulo de elasticidade em flexão estática, no sentido paralelo ou perpendicular à orientação predominante dos flocos das faces e oriunda dos tratamentos. Os valores médios dessa propriedade no sentido paralelo e perpendicular à orientação predominante dos flocos das faces foram iguais a $28.850 \mathrm{kgf} / \mathrm{cm}^{2}$ e $23.115 \mathrm{kgf} / \mathrm{cm}^{2}$, respectivamente.

Segundo a norma CSA O437-0/93, o valor mínimo exigido para o painel ser comercializado é de 55.000 $\mathrm{kgf} / \mathrm{cm}^{2}$, no sentido paralelo, e para o sentido perpendicular, o valor é de $15.000 \mathrm{kgf} / \mathrm{cm}^{2}$.

\section{CONCLUSÕES}

A adição de flocos de pinus aos flocos de eucalipto permitiu a fabricação de chapas com densidade média em torno de $0,70 \mathrm{~g} / \mathrm{cm}^{3}$.

A acetilação reduziu a adsorção de água, inchamento em espessura e expansão linear e influenciou positivamente a dureza Janka, em que as maiores médias encontradas foram as das chapas acetiladas.

A expansão linear paralela, das chapas fabricadas com flocos não-acetilados e oriundos da região de Três Marias, foi maior do que aquela das chapas de madeiras oriundas da região de Ponte Alta.

A acetilação diminuiu a resistência à tração perpendicular das chapas feitas com madeira de Eucalyptus grandis, cujo valor foi inferior ao estipulado pela norma CSA O437-0/93 (1993).

R. Árvore, Viçosa-MG, v.30, n.4, p.659-668, 2006
A resistência ao arrancamento de parafuso, o módulo de ruptura (sentido paralelo e perpendicular) e a compressão longitudinal (sentido perpendicular) das chapas fabricadas com madeira de Eucalyptus grandis foram inferiores às das demais chapas.

A resistência à compressão longitudinal das chapas feitas com madeira de Eucalyptus urophylla, oriunda da região de Três Marias, foi inferior à das chapas feitas com a mesma espécie, porém oriundas da região de Ponte Alta.

\section{REFERÊNCIAS BIBLIOGRÁFICAS}

AMERICAN NATIONAL STANDARD - ANS. Mat-formed wood particleboard: specification ANSI/A 208.1.1993. Gaithersburg: National Particleboards Association, 1993. 9p.

\section{AMERICAN SOCIETY FOR TESTING AND MATERIALS - ASTM. Standard test methods for evaluating properties of wood-base fiber and particle panels: ASTM D 1037-91. Philadelphia: 1991. (Annual Book of ASTM Standards).}

ASSOCIAÇÃO BRASILEIRA DE NORMAS TÉCNICAS - ABNT. Chapas de madeira aglomerada - métodos de ensaio. ABNT NBR 14810-3. São Paulo: 2002.

CANADIAN STANDARDS ASSOCIATION CSA. OSB and waferboard. 0437.0 - 93. Ontario: 1993. 18p.

CLOUTIER, A. Oriented strandboard (OSB): raw material, manufacturing process, properties, and uses. In: INTERNATIONAL SEMINAR ON SOLID WOOD PRODUCTS OF HIGH TECHNOLOGY. Belo Horizonte: 1998. p. 173-185.

FEIST, W.C.; ROWELL, R.M.; ELLIS, W.D. Moisture sorption and acelerated weathering of acetylated and methacrylated aspen. Wood and Fiber Science, n. 23, v. 1, p. 128-136, 1991.

GOUVEIA, F. N.; VITAL, B.R.; SANTANA, M. A. E. Avaliação de três tipos de colchão e três níveis de resina fenolica na produção de chapas de partículas orientadas - OSB. Revista Árvore, v. 27 , n. 3, p. 365-370, 2003. 
GOUVEIA, F. N.; SANTANA, M. A.; SOUZA, M. R.Utilização da madeira de Eucalyptus grandis W. Hill ex Maidem e Eucalyptus urophylla S. T. Blake na fabricação de chapas de partículas orientadas (OSB) e não orientadas. Revista Árvore, v. 24, n. 1, p. 7-12, 2000.

HRÁZSKÝ, J.; KRÁL, P. The influence of particle composition in a three-layer particleboard on its physical and mechanical properties. Journal of Forest Science, v. 49, n. 2, p. 83-93, 2003.

IMAMURA, Y. et al. Dimensional stability and biological resistance of particleboard from acetylated abbizia wood particles. Wood Research, n. 76, p. 49-58, 1989.

IWAKIRI, S. et al. Utilização da madeira de eucalipto na produção de chapas de partículas orientadas - OSB.

Cerne, v. 10, n. 1, p. 46-52, 2004.

KELLY, M.W. Critical literature review of relationships between processing parameters and physical properties of particleboard. Madison, Forest Products Laboratory, 1977, p. 64. (Laboratory General Technical Report FPL-10),

MAHLBERG, R. et al. Effect of chemical modification on wood on the mechanical and adhesion properties of wood fiber/polypropylene fiber and polypropylene/vener composites. Holz als Roh und Werkestoff, v. 10, n. 4, p.319-326, 2001.

MALONEY, T. M. Modern particleboard \& dry-process fiberboard manufacturing. San Francisco: Miller Freemann, 1993. 696 p.

MEndes, L. M. Pinus spp. na Produção de painéis de partículas orientadas (OSB). 2001. 156f. Tese (Doutorado em Tecnologia e Utilização de Produtos Florestais) Universidade Federal do Paraná, Curitiba, 2001.

MOslemi, A. A.. Particleboard. Carbondale: Southern Illinois University Press, 1974. v. 1. 243 p.
OKINO,E.Y.A. et al. Resistência físico-mecânica de chaps aglomeradas de bagaço de cana-de-açucar modificado quimicamente. Sciencia Forestalis, n. 52, p. 35-42, 1997.

ROSENQVIST, M. Acetyil group distribution in acetylated wood investigated by microautoradiography. Holzforschung, v. 55, n. 3, p. 270-275, 2001.

ROWELL, W.C. (Ed.) Handbook of wood chemistry and wood composites. Boca Raton, CRC Press, 2005. 487 p.

ROWELL, W.C.; SIMONSON,R.; TILLMAN, A.M. Acetyl balance for the acetylation of wood particles by the simplified procedure.

Holzforchung, v. 44, n. 4, p. 263-269, 1990.

ROWELL, W.C.; YOUNGQUIST, J.A.; SACHS, I.B. Adhesive bonding of acetylated aspen flakes. Part I. Surface changes, hydrophobicity, adhesive penetration, and strength.

International Journal of Adhesion and Adhesives, v. 7, n. 4, p. 183-188, 1987.

VICK, C. B.; ROWELL, R. M., Adhesive bonding of acetylated wood. Wood research bulletin of the wood research Institute, Kyoto: Kyoto University, 1990. p. 131-141.

VITAL,B.R. Métodos de determinação da densidade da madeira. Viçosa, MG: Sociedade de Investigações Florestais, 1984. 21p.

VITAL, B.R.; LEHMANN, W.F.; BOONE, R.S. How species and board densities affect properties of exotic hardwood particleboards. Forest Products Journal, v. 24, n. 12, p. 37-45, 1974.

YOUNGQUIST, J.A. \& ROWELL, R.M.

Adhesive bonding of acetylated aspen flakes. Part III. Adhesion with isocyanates.

International Journal of Adhesion and Adhesives, v. 10, n. 4, p. 273-276, 1990. 
